Supporting Information

\title{
Intrahelical Interactions in $\alpha$-Helical Coiled Coil Determine the Structural Stability of Tropomyosin
}

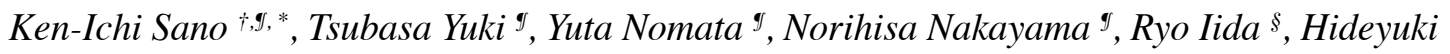 \\ Mitomo *, Kuniharu Ijiro *, Yoshihito Osada
}

\begin{abstract}
'Department of Applied Chemistry, Faculty of Fundamental Engineering, Nippon Institute of Technology, Miyashiro, Saitama 345-8501, Japan

${ }^{9}$ Graduate School of Environmental Symbiotic System Major, Nippon Institute of Technology, Miyashiro, Saitama 345-8501, Japan.

$\S$ Graduate School of Chemical Sciences and Engineering, Hokkaido University, Sapporo 060-8628, Japan

Research Institute for Electronic Science and Global Station for Soft Matter, Global Institution for Collaborative Research and Education (GI-CoRE), Hokkaido University, Sapporo 001-0021, Japan

${ }^{\psi}$ Nano Medical Engineering Laboratory, RIKEN, Wako, Saitama 351-0198, Japan.

*Corresponding Author: E-mail: kisano@nit.ac.jp. Phone: +81-480-33-7725
\end{abstract}

\section{MATERIALS AND METHODS for Supporting Information}

\section{SDS-PAGE analysis}

Uniform 15\% precast gels (Long-life gel, Oriental Instruments, Sagamihara, Japan) were used for SDS-PAGE analysis. The electrophoresis was carried out by constant current at 30 $\mathrm{mA}$ for $50 \mathrm{~min}$. Detection of proteins were done by Coomassie Brilliant Blue staining using Coomassie Tablets, PhastGel Blue R-350 (GE healthcare).

\section{Differential Scanning Calorimetry analysis}

Dialyzed aliquots of proteins were analyzed by DSC using a Microcal VP-Capillary DSC to measure the excess heat capacity of proteins unfolding as a function of temperature. Buffer conditions were indicated in the figure legend, and the concentration of proteins were 1.0 
$\mathrm{mg} / \mathrm{ml}$ and a scan rate of $60{ }^{\circ} \mathrm{C} / \mathrm{h}$. The DSC curves were fitted by nonlinear least squares analysis using the non-two-state model using MicroCal Origin software packaged with DSC.

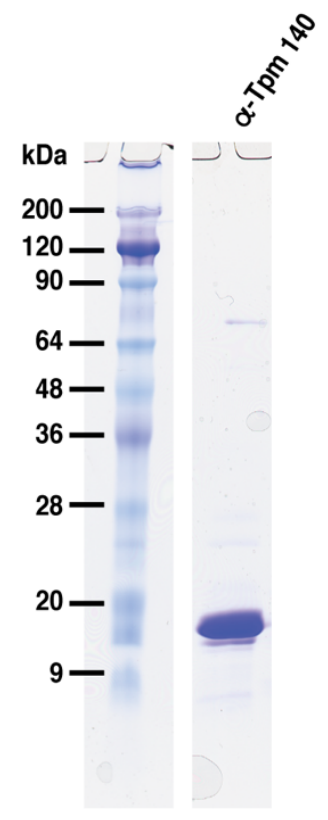

Figure S1. SDS-PAGE of purified $\alpha$-Tpm 140 used in this study.

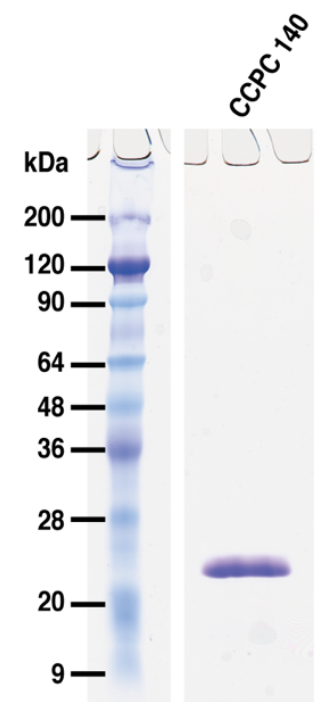

Figure S2. SDS-PAGE of purified CCPC 140 used in this study. 


\section{Results for Supporting Information}

\section{DSC analysis}

CCPC 140 in phosphate buffer exhibit less cooperative melting transitions by $\mathrm{CD}$. We also obtained the thermal denaturation profiles using DSC (Figure S3, Table S1). The DSC denaturation profiles demonstrated that less cooperative melting profiles of CCPC 140 in phosphate buffer on CD is explained by two-step transition states. In contrast to CCPC 140, the denaturation profiles of $\alpha$-Tpm 140 was good fit to a one peak fitting model.
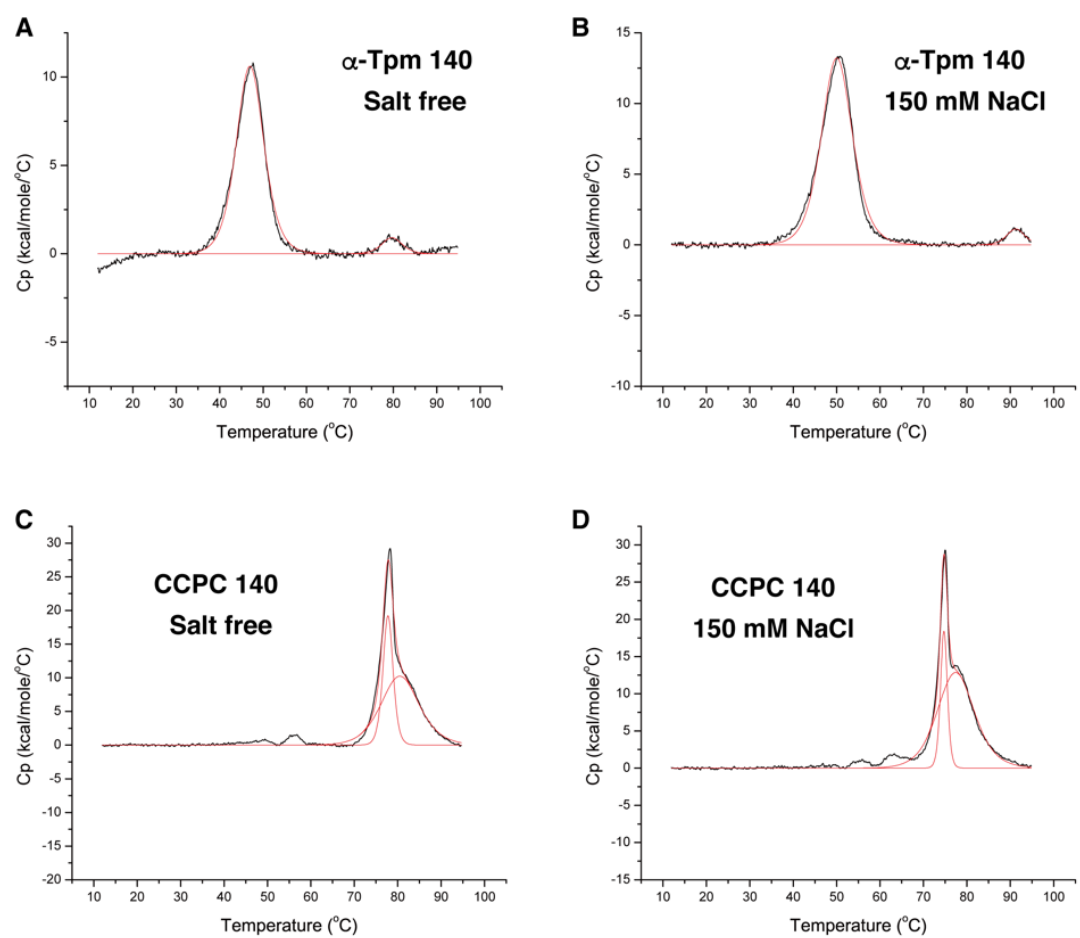

Figure S3. Thermal denaturation profiles of $\alpha$-Tpm 140 (A, B) and CCPC 140 (C, D) by DSC in the presence of $2 \mathrm{mM}$ sodium phosphate buffer, $\mathrm{pH} 8.0$ with $(\mathrm{B}, \mathrm{D})$ or without $(\mathrm{A}, \mathrm{C}) \mathrm{NaCl}$. Black lines represent the profiles of the excess heat capacity buffer-subtracted, concentrationnormalized, and corrected-baseline. Red lines represent the excess heat capacity profiles of the fitting results using the non-two-state model with one peak for $\alpha$-Tpm 140 (A and B) and two peaks for CCPC 140 (C and D). 
Table S1. Summary of DSC analysis

\begin{tabular}{cccc}
\hline $\begin{array}{c}2 \mathrm{mM} \text { phosphate } \\
\text { buffer pH } 8.0\end{array}$ & Salt free & $150 \mathrm{mM}$ & \\
\hline$\alpha-\mathrm{Tpm} 140$ & $47.1^{\circ} \mathrm{C}$ & $50.1^{\circ} \mathrm{C}$ & \\
CCPC 140 & $77.8^{\circ} \mathrm{C}$ & $74.7^{\circ} \mathrm{C}$ & Peak 1 \\
& $80.1^{\circ} \mathrm{C}$ & $77.5^{\circ} \mathrm{C}$ & Peak 2 \\
\hline
\end{tabular}

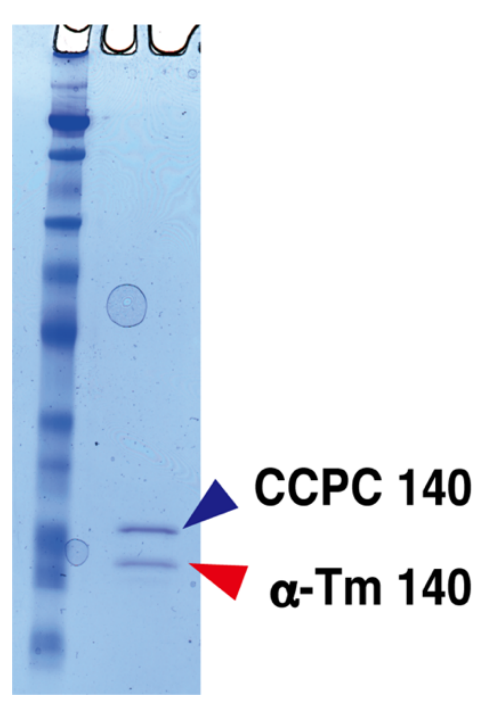

Figure S4. SDS-PAGE of $\alpha$-Tpm 140/CCPC 140 heteromeric species. 

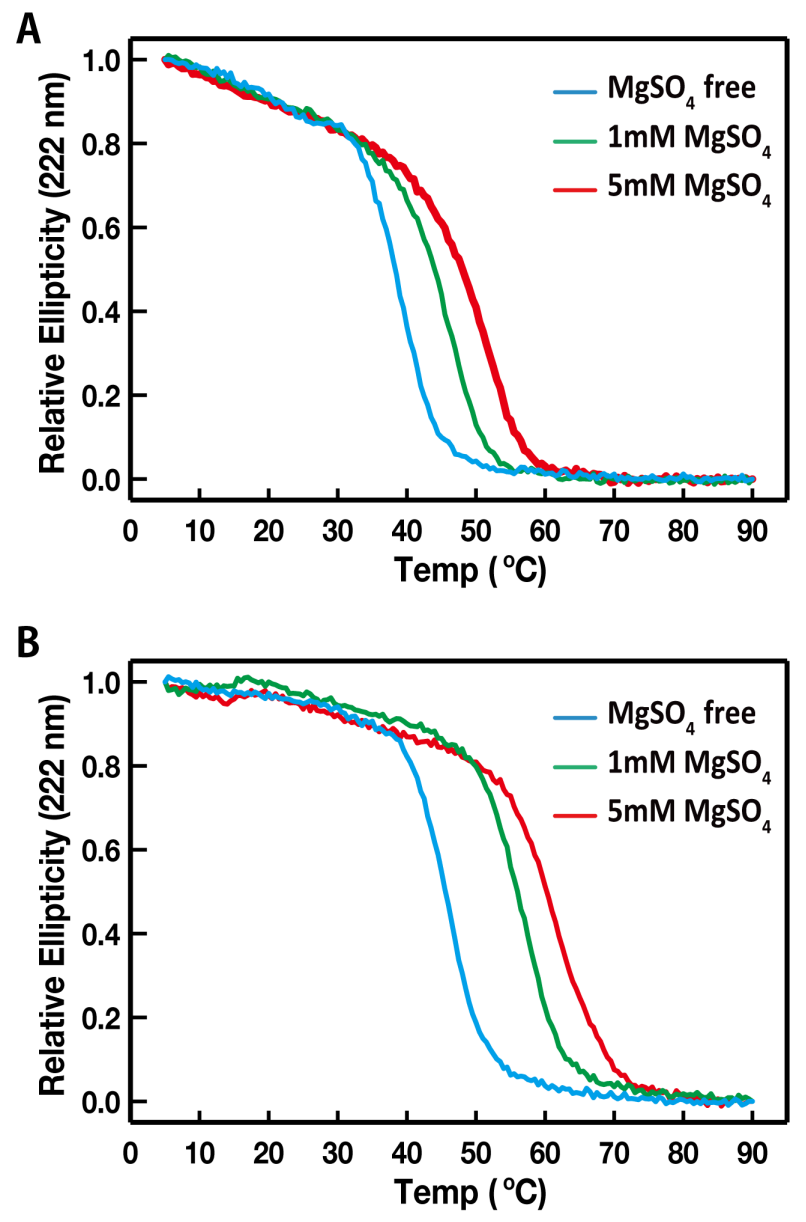

Figure S5. Effect of divalent ions on the thermal melting profiles of $\alpha$-Tpm 140 (A) and CCPC 140 (B) in the presence of $5 \mathrm{mM}$ Tris-buffered solution with or without magnesium sulfate. The light blue line represents without sodium sulfate, green line, and red line represent $1 \mathrm{mM}$ and 5 $\mathrm{mM}$ concentrations of magnesium sulfate, respectively.

Table S2. Tm of $\alpha$-Tpm 140 and CCPC 140 in 5 mM Tris-buffered solution with $\mathrm{MgSO}_{4}$.

\begin{tabular}{clll}
\hline $5 \mathrm{mM}$ Tris-HCI pH 8.0 & Salt free & $1 \mathrm{mM}$ & $5 \mathrm{mM} / \mathrm{MgSO}_{4}$ \\
\hline$\alpha-\mathrm{Tpm} 140$ & $39.3^{\circ} \mathrm{C} *$ & $40.5^{\circ} \mathrm{C}$ & $43.5^{\circ} \mathrm{C}$ \\
$\mathrm{CCPC} 140$ & $46.9^{\circ} \mathrm{C} *$ & $57.5^{\circ} \mathrm{C}$ & $63.0^{\circ} \mathrm{C}$ \\
\hline
\end{tabular}

* Average of 4 independent data sets in Figure 3, 5, 6 and Figure S3. Standard deviation is 0.29 and 0.25 for $\alpha$-Tm 140 and CCPC 140, respectively. 